\title{
Human Papillomavirus Vaccines and the Prevention of Cervical Cancer- The Dawn of a New Era?
}

\author{
a report by \\ Anna Giuliano, PhD \\ Professor, Department of Interdisciplinary Oncology, and Program Leader, Risk Assessment, Detection, and Intervention Program,
} H Lee Moffitt Cancer Center and Research Institute, University of South Florida

DOI: $10.17925 / O H R \cdot 2007.00 .2 .66$

Human papillomavirus (HPV) is thought to be the most common sexually transmitted infection in the US.' It is estimated that as many as $75-80 \%$ of men and women will have contact with HPV at some point in their lives. ${ }^{2}$ In the US, the highest prevalence of HPV is among females between 20 and 24 years of age. Factors that affect the risk of infection include number of sexual partners, marital status, cigarette smoking, and infection with other sexually transmitted diseases (STDs) such as genital herpes. Infection is also common in women following their first sexual encounter. ${ }^{3}$

There are approximately 130 identified types of HPV that can result in genital infection. Low-risk types of HPV such as 6 and 11 cause genital warts. HPV infections are the primary cause of cervical cancer, with HPV types 16 or 18 resulting in approximately $70 \%$ of all cases. A further 11 high-risk HPV types are carcinogenic. ${ }^{4}$ Persistent infection with high-risk HPV types is the major factor in the development of cervical cancer precursor lesions, for example cervical intraepithelial neoplasia (CIN), vaginal intraepithelial neoplasia (VaIN), vulvar intraepithelial neoplasia (VIN), and anal intraepithelial neoplasia (AIN). Worldwide, cervical cancer causes 250,000 deaths per year, hence the emphasis is on preventing the disease through vaccination against HPV infection.

\section{Human Papillomavirus Vaccines}

Two vaccines have been developed to prevent infection with the high-risk HPV types for cervical cancer. The vaccines comprise recombinant viruslike capsules that are morphologically identical to HPV virons, although they contain no actual viral DNA. The first is a quadrivalent vaccine containing HPV high-risk types 16 and 18 and low-risk types 6 and 11, which cause genital warts (Gardasil ${ }^{\circledR}$, Merck Research Laboratories), and the second is a bivalent vaccine containing types 16 and 18 (Cervarix ${ }^{\mathrm{TM}}$, GlaxoSmithKline Biologicals).

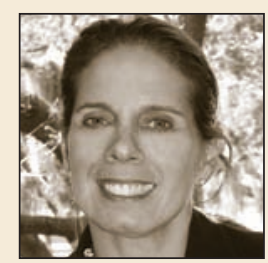

Anna Giuliano, PhD, is a Professor in the Department of Interdisciplinary Oncology and Program Leader for the Risk Assessment, Detection, and Intervention Program at the Moffitt Cancer Center at the University of South Florida. Her research focus is on human papillomavirus (HPV)-related carcinogenesis; her other research interests include cancer epidemiology biomarkers; nutritional supplements and diet as chemopreventive agents; HPV and cervical cancer; breast cancer; and cancer screening. The National Institutes of Health (NIH) has awarded Dr Giuliano a \$10 million grant for a study investigating men's role in the spread of the HPV that causes cervical cancer. This is the largest NIH grant ever awarded to a cancer control and prevention researcher at the Moffitt Cancer Center. Dr Giuliano obtained her $\mathrm{PhD}$ in nutritional biochemistry from Tufts University School of Nutrition in 1990.

E: anna.giuliano@moffit.org
Two multicenter phase III trials testing the efficacy of Gardasil have been completed: Females United to Unilaterally Reduce Endo/Ectocervical Disease I and II (FUTURE I and II). 5,6 FUTURE I included 5,455 women aged 16-22 years, randomized to receive either the quadrivalent vaccine or a placebo. The women's progress was followed for an average of three years after the first dose of the vaccine, which was reported to be $100 \%$ effective at preventing anogenital diseases associated with HPV types 6, 11, 16, and 18.

The second phase III trial, FUTURE II, included 12,167 women between 15 and 26 years of age. Females were randomized to receive either the vaccine or a placebo, and the end-points were CIN grade 2 or 3, adenocarcinoma, or cervical cancer related to HPV types 16 or $18 .{ }^{6}$ In this study, the vaccine was reported to have $100 \%$ efficacy in the prevention of HPV 16/18, CIN 2/3, or adenocarcinoma in situ (AIS), as well as other cervical cancer lesions. Furthermore, the vaccination demonstrated $99 \%$ efficacy in preventing genital warts associated with the same HPV types, and was 100\% effective against vulvar and vaginal pre-neoplastic lesions (VIN 2/3 and ValN 2/3). Thus, widespread immunization of young women with the quadrivalent vaccine would result in a substantial reduction in HPV 16/18-related cervical disease and a reduction in the majority of cases of genital warts.

In the phase II evaluation of the bivalent vaccine, 1,113 women between 15 and 25 years of age were randomized to receive either a three-dose regimen of the vaccine or placebo. ${ }^{7}$ The vaccine was $91.6 \%$ effective at preventing incident infection and $100 \%$ effective at preventing persistent infection with the designated HPV types. The vaccine was generally welltolerated and no serious side effects were reported. A follow-up after 4.5 years showed that more than $98 \%$ seropositivity was maintained for HPV $16 / 18$ antibodies. ${ }^{8}$ The vaccine remained $100 \%$ effective at protecting against CIN and demonstrated cross-reactivity in preventing HPV types 45 and 31 infections. An interim analysis of 15 months of follow-up data from the phase III testing of the bivalent vaccine demonstrated $90.4 \%$ efficacy against development of CIN 2/3 lesions and $80.4 \%$ protection against confirmed persistent HPV 16/18 infection. ${ }^{9}$

Using mathematical modeling, the HPV vaccine appears to be costeffective. ${ }^{10}$ The computer-based mathematical model predicted that a $98 \%$ prevention of HPV type 16/18 would produce a similar reduction in the amount of HPV 16/18-associated cervical cancers and a 51\% decrease in the total number of cases of cervical cancer.

\section{Target Population}

Data from all clinical trials for the quadrivalent vaccine demonstrate that the vaccine will not be optimally effective in women who have previously been 
infected with HPV types 6/11/16/18. Efficacy was much lower (44\%) in women who had an HPV 16/18-related CIN or an infection with either type. ${ }^{6}$ However, those who have been infected with one or more of the vaccine-related types would still remain protected from diseases caused by any of the other HPV types in the vaccine. Thus, it is recommended that the vaccinations be targeted at women prior to sexual initiation to maximize the clinical impact.

From a public health perspective, the vaccination of men alongside that of women should provide further protection within the population.

From a public health perspective, the vaccination of men alongside that of women should provide further protection within the population. In the UK, the vaccine is licensed for use in girls and boys nine to 15 years of age and women 16-26 years of age, hence men over the age of 16 years may receive the vaccine only off-label. In 2006, the US Food and Drug Administration (FDA) approved the quadrivalent vaccine for use solely in females nine to 26 years of age. The vaccine is not approved for males of any age in the US. However, a study is currently under way to determine its safety and efficacy profile in males.

\section{Implementation of the Vaccine}

There is the widespread use of Papanicolaou (Pap) smears in developed countries. Since its introduction, the Pap smear has prevented the development of approximately $50 \%$ of cervical cancer cases worldwide. The quadrivalent vaccine will prevent only $70 \%$ of infections that cause cancer, and a large number of women over the age of 26 years will not have been vaccinated; hence, for the foreseeable future the Pap test will continue to be part of national programs to prevent cervical cancer, even for women who have been vaccinated.

The quadrivalent HPV vaccine is anticipated to cost approximately $\$ 360$ for the three-dose series. While the majority of females eligible to receive the vaccine will be covered by private medical insurance, it is estimated that up to $12 \%$ of girls nine to 18 years of age and $29 \%$ of women $19-26$ years of age will be uninsured. ${ }^{11}$ There are several public funding schemes set up to provide uninsured children with financial support for the vaccine, e.g. the Vaccines for Children program, which covers all vaccines for children who are Medicaid-eligible, uninsured, American Indian or Alaska Native, or underinsured, and the Immunization Grant Program, which awards grants to state, local, and territorial public health agencies. ${ }^{12}$ This will enable the vaccination program to cover a large majority of the target population.

In developing countries, cervical cancer is one of the leading causes of cancer deaths among women, Therefore, the implementation of a vaccination program would have real potential to save lives. National strategies to deliver HPV vaccines in several developing countries are under review and discussion and will likely require public-private partnerships.
For the vaccine to be successful it needs to be cost-effective. A study in Australia examined the cost-effectiveness of adding the HPV vaccine to the existing Australian National Screening Program. ${ }^{13}$ Results were analyzed based on administering a vaccine that prevents $100 \%$ of HPV 16/18 type infections to 12-year-old schoolgirls, the end-points being costs per life-year and quality-adjusted life-year saved. It found that the addition of the vaccine was a cost-effective way to reduce cervical cancer. The introduction in Canada of the HPV vaccination also led to a decrease in cervical cancer. ${ }^{14}$ The main advantage of the vaccine was identified as a reduction of mortality from cervical cancer.

\section{Vaccine Controversies}

The HPV vaccine has encountered several difficulties. The first and most important is the general lack of public awareness and knowledge that cervical cancer and cervical dysplasia are caused by infections with HPV, an infection that can be transmitted through skin-skin contact. It is estimated that only $40 \%$ of women are aware of the association of HPV virus with cervical cancer. ${ }^{15}$ One of the biggest public misconceptions has been that immunizing pre-teens against an STD will encourage promiscuity. However, this is considered by most public health experts to be unlikely. Unwanted pregnancy and AIDS are more likely than the threat of cancer, and the distribution of condoms and sexual education regarding these matters has not increased sexual activity in adolescence. Interestingly, a study from the University of Pennsylvania demonstrated that comprehensive sex education delayed initiation of sex, decreased the incidence of unprotected sex, and reduced the number of sexual partners. ${ }^{16}$

Public knowledge of the safety of the

human papillomavirus vaccine, as

determined in recent clinical trials, will

increase the level of parental consent to

the vaccination program.

To date, Virginia and the District of Columbia have school entry requirements for mandatory vaccination with the HPV vaccine that should soon take effect, and both have an opt-out option for parents who have medical, moral, or religious opposition. There has been increasing parental concern over the safety of the vaccine and many parents refuse to allow administration of the vaccine to their children. ${ }^{17}$ Public knowledge of the safety of the HPV vaccine, as determined in recent clinical trials, will increase the level of parental consent to the vaccination program. Parents with the belief that their child will remain abstinent until marriage view the vaccinations as unnecessary. In the past an abstinence-only sexual education has not been successful at delaying the age of sexual initiation. ${ }^{18}$ The Centers for Disease Control and Prevention (CDC) has estimated that by 15 years of age $13 \%$ of girls are sexually active, and by 17 years of age the figure rises to $43 \% .{ }^{19}$ School-based vaccination programs are important for providing protection from HPV to those most at risk. For the successful implementation of the vaccine, more information needs to be provided to the public on the risks of HPV-related cervical cancer and the benefits stemming from the vaccine. 


\section{Gynecological Cancer}

Summary

HPV is the most common sexually transmitted infection in the US. HPV types 16 and 18 are the most common precursors for cervical cancer, and HPV types 6 and 11 are common causes of genital warts. Persistent infection with highrisk HPV types is the most important factor in the development of cervical cancer precursor lesions. Two vaccines have been developed against HPV: a quadrivalent vaccine comprising types $6,11,16$, and 18, and a bivalent vaccine including types 16 and 18 . The quadrivalent vaccine has been successful at preventing cervical cancer in several clinical trials and was FDAapproved in 2006 to be administered to females nine to 26 years of age. However, the planned introduction of the vaccination has not met with universal welcome. Many parents believe that its introduction will lead to sexual promiscuity. To overcome this obstacle, education on the risks of the disease and benefits of the vaccine need to be presented more effectively.
1. Weinstock $H$, Berman $S$, Cates W, Sexually transmitted infections in American youth: incidence and prevalence estimates, 2000, Perspect Sex Reprod Health, 2004;36:6-10.

2. Koutsky LA, Galloway DA, Holmes KK, Epidemiology of genital human papillomavirus infection, Epidemiol Rev, 1988;10: 122-63.

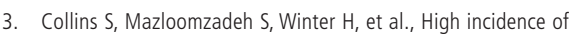
cervical human papillomavirus infection in women during their first sexual relationship, BJOG, 2002;109(1):96-8.

4. IARC Monographs on the Evaluation of Carcinogenic Risks to Humans, 2005, www.monographs.iarc.fr/ENG/Preamble/ amendments.php

5. Garland SM, Hernandez-Avila M, Wheeler CM, et al., Quadrivalent vaccine against human papillomavirus to prevent anogenital disease, N Engl J Med, 2007;356:1928-43.

6. The FUTURE Study Group, Quadrivalent Vaccine against Human Papillomavirus to Prevent High-Grade Cervical Lesions, N Engl J Med, 2007;356:1915-27.

7. Harper DM, Franco EL, Wheeler C, et al., Efficacy of a bivalent $L 1$ virus-like particle vaccine in prevention of infection with human papillomavirus types 16 and 18 in young women: a randomised controlled trial, Lancet, 2004:364(9447):1757-65.
8. Harper DM, Franco EL, Wheeler CM, et al., Sustained efficacy up to 4.5 years of a bivalent L1 virus-like particle vaccine against human papillomavirus types 16 and 18: follow-up from a randomised control trial, Lancet, 2006:367(9518):1247-55.

9. Paavonen J, Jenkins D, Bosch FX et al., Efficacy of a prophylactic adjuvanted bivalent L1 virus-like-particle vaccine against infection with human papillomavirus types 16 and 18 in young women: an interim analysis of a phase III doubleblind, randomised controlled trial, Lancet, 2007;369(9580): 2161-70. Erratum in: Lancet, 2007;370(9596):1414.

10. Goldie SJ, Grima D, Kohli M, et al., A comprehensive natural history model of HPV infection and cervical cancer to estimate the clinical impact of a prophylactic HPV-16/18 vaccine, Int Cancer, 2003;106:896-904.

11. Kaiser Commission on Medicaid and the Uninsured, The Medicaid Resource Book, July 2002.

12. Hinman A, W Orenstein, L Rodewald, Financing Immunization in the United States, Clin Infect Dis, 2004;38:1440-46.

13. Kulasingam S, Connelly L, Conway E, A cost-effectiveness analysis of adding a human papillomavirus vaccine to the Australian National Cervical Cancer Screening Program, Sex Health, 2007:4(3):165-75.
14. Brisson M, Van de Velde N, De Wals N, The potential costeffectiveness of prophylactic human papillomavirus vaccines in Canada, Vaccine, 2007;25(29):5399-5408

15. Tiro J, Meissner H, Kobrin S, et al., What Do US Women Know About HPV and Cervical Cancer?, Abstract from the American Association of Cancer Research 'Frontiers in Cancer Prevention Research' Conference, November 2006.

16. Bleakley A, Hennessy M, Fishbein M, Public opinion on sex education in US schools, Arch Pediatr Adolesc Med, 2006;160: $1151-6$.

17. Freed G, Clark S, Hibbs B, et al., Parental Vaccine Safety Concerns: The Experiences of Pediatricians and Family Physicians, Am J Prev Med, 2004;26(1):11-14.

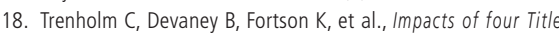
$V$, Section 510 abstinence education programs: final report, Princeton, New Jersey: Mathematica Policy Research, April 2007. Accessed April 19, 2007, www.mathematica$\mathrm{mpr}$. com/publications/PDFs/ impactabstinence.pdf

19. Dailard C, Legislating against arousal: the growing divide between federal policy and teenage sexual behavior, Guttmacher Policy Rev, 2006;9:12-16.

\section{Gynecological Cancer-At a Glance}

- As of January 2008, approximately 20 million Americans are infected with human papillomavirus (HPV), with a further 6.2 million people becoming infected each year.

- In $90 \%$ of cases, the body's immune system clears HPV infection naturally within two years for both high-risk (cancer-causing) and low-risk (wart-causing) types.

- Over $80 \%$ of women newly diagnosed with cervical cancer are from developing countries. Of the 260,000 deaths from cervical

Ovarian Cancer Incidence Rates Per Anum in the US by Race

\section{Per 100,000 women}

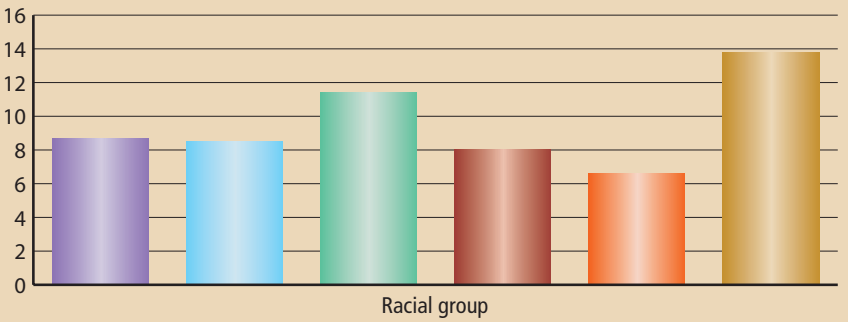

11 All races |Whites || Black II Asian/Pacific Islander II American Indian/Alaska Native* II Hispanic**

* Based on contract health delivery area counties. ** Excludes deaths in Minnesota, New Hampshire, and Dakota.

\section{Ovarian Cancer Death Rates Per Anum in the US by Race}

Per 100,000 women

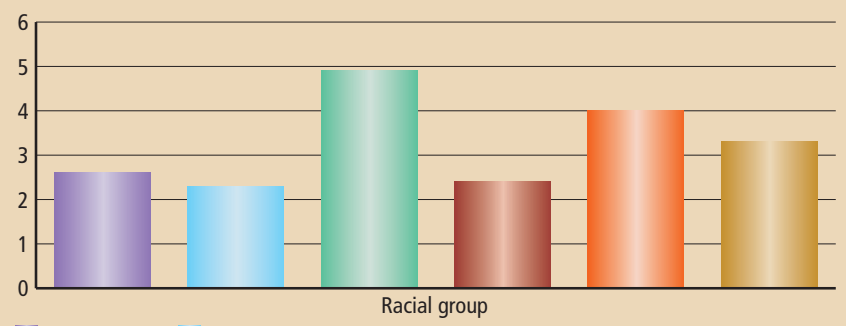

II All races Whites || Black || Asian/Pacific Islander II American Indian/Alaska Native* II Hispanic**

* Based on contract health delivery area counties. ** Excludes deaths in Minnesota, New Hampshire, and Dakota.

cancer worldwide in 2005, approximately 95\% were in developing countries.

- Hispanic women are most at risk for contracting cervical cancer, with double the rate of non-Hispanic white women.

- A recent University of Hawaii report suggested that for each year a woman takes an oral contraceptive, the risk of ovarian cancer is reduced by an average of approximately $5 \%$. The reasons behind this are unclear.

Sources: Centers for Disease Control and Prevention, WHO Comprehensive Cervical Cancer Control, American Cancer Society, Reuters Health. 


\section{GARDASIL help protect a generation of girls and young women}

The only quadrivalent vaccine that prevents disease caused by significant human papillomavirus (HPV) types: 6, 11, 16, and 18

\section{- CERVICAL CANCER \\ - CERVICAL DYSPLASIA \\ - GENITAL WARTS}

GARDASIL is a vaccine indicated in girls and women 9 to 26 years of age for the prevention of cervical cancer, precancerous or dysplastic lesions, and genital warts caused by HPV Types 6, 11, 16, and 18.

A complete vaccination regimen of GARDASIL consists of a 3-dose schedule. See Dosage and Administration section of the Prescribing Information.

\section{Select safety information}

GARDASIL is contraindicated in individuals who are hypersensitive to the active substances or to any of the excipients of the vaccine.

GARDASIL does not substitute for routine cervical cancer screening, and women who receive GARDASIL should continue to undergo screening per standard of care.

Vaccination with GARDASIL may not result in protection in all vaccine recipients.

GARDASIL is not intended to be used for treatment of active genital warts; cervical cancer; cervical intraepithelial neoplasia, vulvar intraepithelial neoplasia, or vaginal intraepithelial neoplasia.

The vaccine-related adverse experiences that were observed among recipients of GARDASIL at a frequency of at least $1.0 \%$ and greater than placebo were pain, swelling, erythema, fever, nausea, pruritus, and dizziness.

Please read Brief Summary of Prescribing Information on adjacent page.

- Vaccinate today-girls and young women 9 to 26 years of age
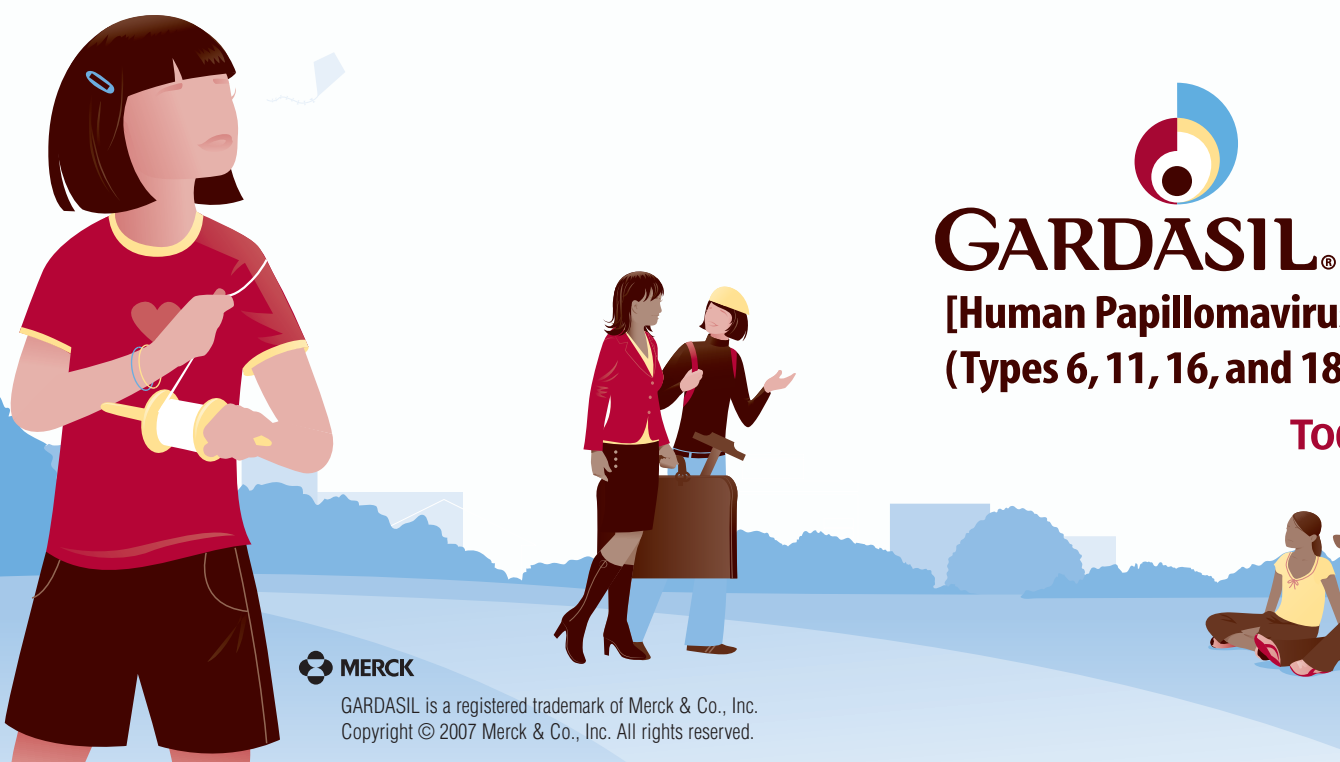

[Human Papillomavirus Quadrivalent

(Types 6, 11, 16, and 18) Vaccine, Recombinant]

Today, you can do more

GARDASIL is a registered trademark of Merck \& $\mathrm{C} 0$., Inc. Copyright $@ 2007$ Merck \& Co., Inc. All rights reserved. 